\title{
Scaling of electromagnetic transducers for shunt damping and energy harvesting
}

Stephen J. Elliott ${ }^{\mathrm{a}}$

Michele Zilletti ${ }^{\text {b }}$ sje@isvr.soton.ac.uk

michele.zilletti@uniud.it

${ }^{a}$ Institute of Sound and Vibration Research, Highfield, Southampton SO171BJ, UK

${ }^{\mathrm{b}}$ DIEGM, Università degli Studi di Udine,Via delle Scienze, 208, 33100 Udine, Italy

Keywords: energy harvesting, shunt damping, electromagnetic transducers

\begin{abstract}
In order for an electromagnetic transducer to operate well as either a mechanical shunt damper or as a vibration energy harvester, it must have good electromechanical coupling. A simple two-port analysis is used to derive a non-dimensional measure of electromechanical coupling, which must be large compared with unity for efficient operation in both of these applications. The two-port parameters for an inertial electromagnetic transducer are derived, from which this non-dimensional coupling parameter can be evaluated. The largest value that this parameter takes is approximately equal to the square of the magnetic flux density times the length of wire in the field, divided by the mechanical damping times the electrical resistance. This parameter is found to be only of order of one for laboratory devices that weigh about $1 \mathrm{~kg}$, and so such devices are generally not efficient, within the definition used here, in either of these applications. The non-dimensional coupling parameter is found to scale in approximate proportion to the device's characteristic length, however, and so although miniaturised devices are less efficient, much greater efficiency can be obtained with large devices, such as those used to control civil engineering structures.
\end{abstract}

\section{INTRODUCTION}

Electromagnetic transducers can be used for either shunt damping [1,2] or energy harvesting [3, 4]. The performance of such a device in both of these applications is analysed here in terms of its twoport parameters. A single dimensionless parameter is found to govern the efficiency of the device when used for either shunt damping or energy harvesting. The scaling of the parameter with the size of the transducer is then investigated and the result contrasted with the corresponding result for a piezoceramic actuator. 
Assuming that the moving parts of an electrodynamic actuator all vibrate in phase and that it is linear, its response at a single frequency can be completely defined by the two-port network equations $[5,6]$, which may be written as:

$$
\begin{gathered}
u=Z_{e b} i+T v \\
f=-T i+Z_{m o} v,
\end{gathered}
$$

where $u, i, f$ and $v$ are the voltage across the device's terminals, the current through the device, the force generated by the device and its velocity. $Z_{e b}$ is thus the device's blocked electrical impedance, $Z_{m o}$ is its open circuit mechanical impedance and $T$ its transduction coefficient, each of which are, in general, complex, frequency-dependant parameters.

Figure 1 shows a block diagram of the transducer, represented in terms of the two-port parameters. There are two circuits, representing either the mechanical or electrical responses, each coupled via a generator representing the electromechanical coupling.

When the base of the transducer is fixed and the force acts on the moving mass, an electromagnetic actuator can be idealised as on the left hand side of Figure 2. This model is widely used to represent the dynamics of shaker mounted on a rigid base, for example. Assuming the mechanical parts move as a single degree of freedom system, then the two port parameters for the transducer in this case are:

$$
\begin{gathered}
Z_{e b}=R_{e}+j \omega L_{e}, \\
Z_{m o}=j \omega M+\frac{K}{j \omega}+R_{m}, \\
T=B l .
\end{gathered}
$$

where $R_{e}$ and $L_{e}$ are the electrical resistance and inductance of the coil $M, K$ and $R_{m}$ are the mass, stiffness and mechanical resistance of the moving parts, $B$ is the flux density and $l$ is the length of wire moving in the field. The electrical and mechanical variables are assumed to be proportional to $e^{j \omega t}$ where $\omega$ is the angular excitation frequency. When operated at its natural frequency, $\omega_{n}=$ $\sqrt{K / M}$, the open circuit mechanical impedance is equal to $R_{m}$ and, since $\omega L_{e}$ is generally much less that $R_{e}$ at this frequency, $Z_{e b}$ is approximately equal to $R_{e}$.

If, however, the electromagnetic transducer is used as an inertial device, so that the force acts on its base and the mass vibrates freely, as on the right hand side of Figure 2, the two port parameters can then be shown to be:

$$
\begin{gathered}
Z_{e b}=R_{e}+j \omega L_{e}+\frac{j \omega(B l)^{2}}{j \omega R_{m}+K-\omega^{2} M}, \\
Z_{m o}=\frac{j \omega M\left(K+j \omega R_{m}\right)}{j \omega R_{m}+K-\omega^{2} M},
\end{gathered}
$$




$$
T=\frac{-\omega^{2} M B l}{j \omega R_{m}+K-\omega^{2} M} .
$$

Figure 3 shows the modulus of these two port parameters, as a function of frequency, for both cases with the assumed parameters listed in Table 1.

If the inertial device is operated above its natural frequency, $\omega_{n}$, but below the frequency at which the inductance becomes important, $\omega_{u}=R_{e} / L_{e}$, then $Z_{e b}$ is approximately equal to $R_{e}, Z_{m o}$ is approximately equal to $R_{m}$ and $T$ is approximately equal to $B l$. These approximations hold for well damped devices with $\zeta>1 / 2$. For damping ratio lower than $1 / 2$ the cut off frequency above which these approximations are still valid is approximately given by $\omega_{n} / 2 \zeta$ [7]. For the device whose parameters are listed in Table 1, this frequency range is from approximately $30 \mathrm{~Hz}$ to $1 \mathrm{kHz}$, as confirmed by the results shown in Figure 3.

\section{SHUNT DAMPING AND ENERGY HARVESTING}

When used as either a shunt damper or an energy harvester, an electrical impedance, $Z_{\text {shunt }}$, is connected across the electrical terminals of the transducer, as shown in Figure 4. The difference between the two applications is that for shunt damping the objective is to modify the mechanical response of the device, whereas for energy harvesting the objective is to transfer as much power into the electrical shunt as possible, so that it may be stored and used for other purposes.

\subsection{SHUNT DAMPING}

The voltage across the terminals, $u$, is equal to $-Z_{\text {shunt }} i$, since $i$ is defined to flow onto the transducer in Figure 1. The two-port equations (1) and (2), can then be used to show that the mechanical impedance presented by the transducer in this case is:

$$
Z_{m}=Z_{m o}+\frac{T^{2}}{Z_{e b}+Z_{\text {shunt }}} .
$$

Assuming the actuator is being used in the frequency range where $Z_{e b}$ is resistive, and that the shunt is passive, the greatest increase in $Z_{m}$ will be achieved if the shunt impedance is set to zero, i.e. the device is short circuit. In this case $Z_{m}$ is equal to $Z_{m s}$, given by:

$$
Z_{m s}=Z_{m o}\left(1+\frac{T^{2}}{Z_{m o} Z_{e b}}\right) \text {. }
$$

If the electromagnetic transducer is mass-driven and at resonance, or if it is base-driven in the frequency range discussed in Section 1 , so that $T$ is approximately equal to $B l, Z_{e b}$ is approximately equal to $R_{e}$ and $Z_{m o}$ is approximately equal to $R_{m}$, then we can express the ratio of the short circuit mechanical impedance to the open circuit mechanical impedance as: 


$$
\frac{Z_{m s}}{Z_{m o}}=1+C_{e m}
$$

where we define $C_{e m}$ to be the non-dimensional electromagnetic coupling coefficient given by

$$
C_{e m}=\frac{(B l)^{2}}{R_{m} R_{e}} .
$$

The ratio $Z_{m s} / Z_{m o}$ is a measure of the extent to which shorting an electromagnetic actuator can affect its impedance and hence achieve shunt damping. The variation of $Z_{m s} / Z_{m o}$ with $C_{e m}$ is shown in Figure 5.

It is clear that there will be little shunting effect on the mechanical impedance, if $C_{e m}$ is small compared to unity, but a considerable effect if $C_{e m}$ is large compared with unity. It is possible to synthesise electrical impedances that are not passive with special circuits, in which case the resistive part of the shunt impedance could be negative, cancelling out some of the resistive part of $Z_{e b}$, which would have a greater effect on $Z_{m}$ than just short circuiting [1]. To have a significantly greater effect than just short circuiting, however, most of the resistive part of $Z_{e b}$ would have to be cancelled [2], which makes the stability of the system rather sensitive to changes in $Z_{e b}$ due, for example to variations in temperature.

\subsection{ENERGY HARVESTING}

When used for energy harvesting purposes, we assume that the transducer is subject to a constant excitation velocity, $v$. Since the voltage across the terminals is equal to $-Z_{\text {shunt }} i$, then using equation (1), the current generated is:

$$
i=\frac{-T v}{Z_{e b}+Z_{\text {shunt }}} .
$$

The electrical power transferred to the shunt is:

$$
W_{e}=\frac{1}{2} \operatorname{Re}\left(i^{*} u\right)=\frac{1}{2}|i|^{2} \operatorname{Re}\left\{Z_{\text {shunt }}\right\},
$$

where Re denotes the real part of a complex quantity and * indicates complex conjugate, so that:

$$
W_{e}=\frac{|T|^{2}|v|^{2} \operatorname{Re}\left\{Z_{\text {shunt }}\right\}}{2\left|Z_{e b}+Z_{\text {shunt }}\right|^{2}} .
$$

The harvested power is maximised, in this case where a constant velocity excitation is assumed, if $Z_{\text {shunt }}$ is equal to $Z_{e b}^{*}$. If the mass-driven actuator is driven at resonance or the base-driven actuator is again driven in the frequency range discussed at the end of Section 1 , then $Z_{e b}$ is equal to $R_{e}$, and so for maximum harvested power $Z_{\text {shunt }}$ must also equal $R_{e}$, in which case:

$$
W_{e}=\frac{|T|^{2}|v|^{2}}{8 R_{e}} \text {. }
$$

The mechanical power absorbed by the transducer is: 


$$
W_{m}=\frac{1}{2} \operatorname{Re}\left\{v^{*} f\right\}=\frac{1}{2}|v|^{2} \operatorname{Re}\left\{Z_{m}\right\},
$$

where the mechanical impedance of the transducer is, in general, given by equation (9). If $Z_{e b}$ and $Z_{\text {shunt }}$ are again assumed to be equal to $R_{e}$, then using equation (9) the mechanical power is given by:

$$
W_{m}=\frac{1}{2}|v|^{2} \operatorname{Re}\left\{Z_{m o}+\frac{T^{2}}{2 R_{e}}\right\} .
$$

Although there are many figures of merit that could be used for such transducers, we define the power harvesting efficiency, $E$, as the ratio of the electrical power harvested to the mechanical power supplied, so that:

$$
E=\frac{W_{e}}{W_{m}}=\frac{|T|^{2}}{\operatorname{Re}\left\{Z_{m o}+T^{2} / 2 R_{e}\right\} 4 R_{e}} .
$$

If the electromagnetic transducer is mass-driven at resonance, or it is base-driven in the frequency range discussed in Section 1, then $T$ is approximately equal to $B l$ and $\operatorname{Re}\left\{Z_{m o}\right\}$ to $R_{m}$, so that:

$$
E=\frac{(B l)^{2}}{4 R_{m} R_{e}+2(B l)^{2}}=\frac{C_{e m}}{4+2 C_{e m}},
$$

where $C_{e m}$ is again the electromagnetic coupling coefficient given by equation (12) [4]. The variation of $E$ with $C_{e m}$ is also shown in Figure 5.

The efficiency of the energy harvesting device is clearly low if $C_{e m}$ is small compared to unity, but reaches a maximum value of $50 \%$ when $C_{e m}$ is large compared with unity. In this case the electromagnetic coupling is strong, little power is dissipated in the mechanical parts of the transducer and an equal power is dissipated in the internal electrical resistance and harvested on the external shunt.

\section{SCALING OF COUPLING COEFFICIENT WITH DEVICE SIZE}

If the cross-section area of the wire used in the coil of the electromagnetic actuator is $A_{w}$ and the resistivity of the material is $\eta$, then the resistance of the coil is given by:

$$
R_{e}=\eta \frac{l}{A_{w}}
$$

where $l$ is the length of wire in the coil, which is given, approximately, by

$$
l=\frac{V_{c}}{A_{w}}
$$

and $V_{c}$ is the volume of the coil. The quantity $l^{2} / R_{e}$, which appears in the expression for the electromagnetic coupling coefficient, $C_{e m}$, in equation (12), is thus given by 


$$
\frac{l^{2}}{R_{e}}=\frac{V_{c}}{\eta}
$$

which is independent of the number of turns in the coil. The resistivity of the coil material does not depend of the scale of the transducer, but the volume of the coil clearly scales as the cube of characteristic length of the transducer, $L$, so that it scales as $\left[L^{3}\right]$ in the notation used in reference [810], for example. The magnetic flux density $B$ is assumed to be saturated in a well-designed transducer, so this depends on the properties of the materials that the transducer is made from, but not its dimensions. Finally, the scaling of the mechanical resistance, $R_{m}$, depends on the detailed mechanism of mechanical damping in the transducer and we will infer this from experimental data. Table 2 lists the parameters of a number of different electromagnetic actuators, including their nondimensional coupling coefficient. The variation of this parameter with the mass of the transducer is plotted in Figure 6. A least square fit to the data shown in Figure 7 suggests that the electromagnetic coupling coefficient scales as the mass raised to the power of about 0.35 . Since the mass of the device clearly scales as $\left[L^{3}\right]$, the overall electromagnetic coupling coefficient, $C_{e m}$, given by $(B l)^{2} / R_{e} R_{m}$, thus must scale approximately in proportion to the characteristic length of the transducer, as [L]. Assuming that $(B l)^{2} / R_{e}$ still scales as $\left[L^{3}\right]$, this result implies that $R_{m}$ scales as about $\left[L^{2}\right]$ for these devices, rather than in proportion to [L], as suggested in [11]. A reasonable fit to the data is thus that electromagnetic coupling coefficient is given by:

$$
\frac{(B l)^{2}}{R_{m} R_{e}} \approx\left(\frac{M_{T}}{M_{0}}\right)^{.35},
$$

where $M_{T}$ is the total mass of the transducer and $M_{0}$ is a reference mass, given from Figure 6 as about $0.6 \mathrm{~kg}$.

This scaling of the coupling coefficient for electromagnetic transducers is in contrast to the equivalent quantity for piezoelectric transducers, as discussed in the Appendix, which depends only on the material properties and is thus, to a first approximation, independent of $L$. It is shown in the Appendix that the equivalent coupling coefficient for piezoelectric transducers $C_{\text {piezo }}$, is related to the piezoelectric coupling factor, $k_{\text {piezo }}^{2}$, by the expression:

$$
C_{\text {piezo }}=\frac{k_{\text {piezo }}^{2}}{1-k_{\text {piezo }}^{2}},
$$

We can thus define a coupling factor for a generic electromagnetic transducer, $k_{e m}^{2}$, as a function of the electromagnetic coupling coefficient, by analogy with the piezoelectric case as:

$$
k_{e m}^{2}=\frac{C_{e m}}{1+C_{e m}},
$$


which varies from 0 to 1 as $C_{e m}$ increase from 0 to $\infty$. Figure 7 shows the variation of $k_{e m}^{2}$, with the actuator mass, assuming that $C_{e m}$ scales with mass as in equation (24), with a constant of proportionality obtained from Figure 6. Also shown on this graph is the value of $k_{\text {piezo }}^{2}$ for a PZT transducer, which is assumed to be independent of actuator mass, as discussed in the Appendix. It can be seen that $k_{e m}^{2}$ is smaller than $k_{\text {piezo }}^{2}$ for actuator masses below about $6 \mathrm{~kg}$, whereas it is larger than $k_{\text {piezo }}^{2}$ for larger devices.

In order to assess the practicability of larger electromagnetic devices, it is also useful to estimate the scaling of some of its other properties with device size. Gardonio et al.[10], for example, show that the devices natural frequency, $\omega_{n}=\sqrt{K / M}$, scales as $\left[L^{-1}\right]$ and that the static displacement due to gravity, $M g / K$, where $g$ is the acceleration due to gravity, scales as $\left[L^{2}\right]$. As the actuator gets larger, its natural frequency tends to fall, as expected, but of more concern is that its static displacement due to gravity will become large rather quickly. This is not a concern if the device can be used horizontally, but if used vertically this static displacement can become larger than the size of the device itself and may ground the inertial mass. Although various self-levelling strategies can be adopted [12] these often require external power, which compromises energy harvesting performance, and so the static displacement may limit the size of the device that can be used in practice.

The operating frequency range of the inertial device has also been assumed to be from somewhat above the natural frequency, $\omega_{n}$, to an upper frequency, $\omega_{u}$, when the reactance of the device's inductance is equal to its electrical resistance, given by $R_{e} / L_{e}$. The dependence of $R_{e}$ on the geometric and electrical properties of the coil is given by equation (21). If the coil has a radius $r$ and height $h$, then since $l$ is approximately equal to $\pi r N$, where $N$ is the number of turns, and $A_{w}$ is equal to $r h / N$, then:

$$
R_{e} \approx \eta \frac{\pi N^{2}}{h},
$$

where $\eta$ is the resistivity of the wire. The electrical inductance can also be approximated by that of a solenoid coil:

$$
L_{e}=\mu \frac{A_{s} N^{2}}{h}
$$

where $\mu$ is the magnetic permeability and $A_{s}$ is the area of the solenoid. $A_{s}$ is equal to $r h$ in this case so the inductance is approximately given by:

$$
L_{e} \approx \mu N^{2} r
$$

The upper limiting frequency $\omega_{u}$, given by $R_{e} / L_{e}$ is thus equal to: 


$$
\omega_{u}=\frac{\eta}{\mu} \frac{\pi}{r h}
$$

which is independent of the number of turns, $N$, but scales as $\left[L^{-2}\right]$.

The frequency range of operation of an inertial device is assumed to be above $\omega_{n}$, which scales as

$\left[L^{-1}\right]$, but below $\omega_{u}$, which scales as $\left[L^{-2}\right]$, and this range thus becomes smaller as the size of the device is scaled up. The upper frequency range can be extended somewhat by using electrical circuit with a negative inductance to cancel the electrical inductance of the device. Similar circuits, but with negative capacitance are currently used to increase the frequency range over which shunt dampers using piezoelectric actuators can be used [13]. There are limitations to the use of such circuits, however, since the magnetic behaviour can be more complicated than that leading to the linear model in equations (3), (4) and (5), with parameters that can be frequency and amplitude dependent.

\section{CONCLUSIONS}

The efficiency of an electromagnetic transducer when used as either a shunt damper or an energy harvester has been shown, under realistic circumstances, to depend on a non-dimensional electromagnetic coupling coefficient, equal to $(B l)^{2} / R_{e} R_{m}$. This coupling coefficient, which varies from zero for a poorly coupled transducer to infinity for a well coupled device, is shown to increase with the size of the electromagnetic transducer, approximately in proportion to its characteristic length. This is in contrast to a piezoelectric transducer, for which the corresponding coupling coefficient is independent of the size of the device. The coupling coefficient is related to the coupling factor widely used to characterise piezoelectric transducers, which varies from zero to unity.

Although an electromagnetic transducer can be used as either a shunt damper or energy harvester, there may also be applications where it is used simultaneously for both purposes. The harvested electrical energy could potentially be used to power a self-tuning shunt damper, for example, to give an entirely autonomous device $[14,15]$ which may even give enough power to drive a digital controller [13].

It has been demonstrated that, because of the scaling of the coupling coefficient with the transducer's size, such systems may be most efficient for electromagnetic devices weighing many kilogrammes, as used in civil engineering applications for example, but would not bevery efficient for miniaturised device, even though it is such devices that have been most widely studied for energy harvesting. 


\section{ACKNOWLEDGEMENTS}

This work is based on a paper presented at the ISMA conference in 2012 [16] and grew out of a previous conference paper on the possibilities of combined energy harvesting and shunt damping [14] with a wider authorship. We would like to thank Nicolas Loix of Micromega for supplying some of the parameters for their actuators and also Paul Reynolds and Donald Nyawako at the University of Sheffield for their measurements on the APS 400 actuator that allowed the parameters used here to be identified.

\section{APPENDIX A}

In order to compare the analysis above with that for piezoelectric transducers, we can write the two port equations for a single-layered piezoelectric transducer, of area $A$ and thickness $t$ as:

$$
\begin{gathered}
q=C_{e} u+D w \\
f=-D u+S_{m} w
\end{gathered}
$$

where $q, u, f$ and $w$ are the charge, voltage, force and extension of the actuator, and [17]:

$$
\begin{gathered}
C_{e}=\frac{\epsilon^{T} A\left(1-k^{2}\right)}{t} \\
D=\frac{d_{33} A}{S^{E} t} \\
S_{m}=\frac{A}{S^{E} t}
\end{gathered}
$$

where $\epsilon^{T}, S^{E}, d_{33}$ and $k^{2}$ are the permittivity, compliance, piezoelectric constant and electromechanical coupling factor of the piezoelectric material.

If the piezoelectric transducer is open circuit then for harmonic excitation the current, given by $j \omega q$ is equal to zero, so that unless $\omega$ is zero, then $q$ must also equal zero, and so the open circuit stiffness is equal to:

$$
\left.\frac{f}{w}\right|_{\text {open circuit }}=S_{m}\left(1+\frac{D^{2}}{S_{m} C_{e}}\right),
$$

where $S_{m}$ is the mechanical stiffness if the device is short circuited.

The stiffness, and hence the impedance, of a piezoelectric transducer is thus greater when it is open circuit than when it is short circuit, in contrast to an electromagnetic transducer, whose impedance is greater when short circuit than when it is open circuit, as shown in equation (11). Another difference is that whereas an electromagnetic device, being velocity controlled, can add damping when short circuit, the piezoelectric device, being displacement controlled, is dominated by its stiffness when either open or short circuit and so requires a resistive element in the shunt to provide 
a damping impedance. Nevertheless, we can define an electromechanical coupling coefficient for this piezoelectric case, by taking the analogy between equation (A.6) and equation (10), as:

$$
C_{\text {piezo }}=\frac{D^{2}}{S_{m} C_{e}}
$$

which can also be written, using the definitions in equations (A.3) to (A.5), as:

$$
C_{\text {piezo }}=\frac{k_{\text {piezo }}^{2}}{1-k_{\text {piezo }}^{2}}
$$

where

$$
k_{\text {piezo }}^{2}=\frac{d_{33}^{2}}{S^{E} \epsilon^{T}}
$$

is the electromechanical coupling factor of the piezoelectric material [17], which can vary from 0 to 1, but is typically 0.1 for PVDF and 0.7 for PZT. As $k_{\text {piezo }}^{2}$ varies from 0 to 1 , the electromechanical coupling coefficient in this case $C_{\text {piezo }}$, varies from 0 to $\infty$, being about 0.1 for PVDF transducer and 2.3 for PZT devices.

Since $k_{\text {piezo }}^{2}$ depends only on the piezoelectric material, and not on the transducer dimensions, then $k_{\text {piezo }}^{2}$ and hence $C_{\text {piezo }}$ for a piezoelectric transducer, does not depend on the size of the transducer, in contrast to the case for the electromagnetic actuator above.

\section{REFERENCES}

[1] A.J. Fleming, S.O.R. Moheimani, Inertial vibration control using a shunted electromagnetic transducer, IEEE/ASME Transactions on Mechatronics, 11 (2006) 84-92.

[2] C. Paulitsch, P. Gardonio, S.J. Elliott, Active Vibration Damping Using an Inertial, Electrodynamic Actuator, ASME Journal of Vibration and Acoustics, 129 (2007) 39-47.

[3] P. Glynne-Jones, M.J. Tudor, S.P. Beeby, N.M. White, An electromagnetic, vibration-powered generator for intelligent sensor systems, Sensors and Actuators A: Physical, 110 (2004) 344-349.

[4] K. Nakano, S.J. Elliott, E. Rustighi, A unified approach to optimal conditions of power harvesting using electromagnetic and piezoelectric transducers, Smart Materials and Structures, 16 (2007) 948.

[5] F.V. Hunt, Electroacoustics The Analysis of Transduction and Its Historical Background, Wiley and Sons, New York, 1954.

[6] S. Roundy, On the Effectiveness of Vibration-based Energy Harvesting, Journal of Intelligent Material Systems and Structures, 16 (2005) 809-823.

[7] M. Zilletti, Self tuning vibration absorbers, in: ISVR, University of Southampton, Southampton, UK, 2012.

[8] M.J. Madou, Fundamentals of Microfabrication: The Science of Miniaturization, Second Edition, 1st Edition ed., CRC Press, Boca Raton, 1997.

[9] C. Gonzalez Diaz, C. Paulitsch, P. Gardonio, Active damping control unit using a small scale proof mass electrodynamic actuator, The Journal of the Acoustical Society of America, 124 (2008) 886-897.

[10] P. Gardonio, C. González Díaz, Downscaling of proof mass electrodynamic actuators for decentralized velocity feedback control on a panel, Smart Materials and Structures, 19 (2010) 025004 . 
[11] J. Peirs, Design of micromechatronic systems: scale laws, technologies and medical applications, in, Katholieke Universiteit Lueven, Phd Thesis, 2001.

[12] L. Benassi, S.J. Elliott, Active vibration isolation using an inertial actuator with local displacement feedback control, Journal of Sound and Vibration, 278 (2004) 705-724.

[13] S. Takeuchi, K. Makihara, J. Onoda, Reliable and Evolvable Vibration Suppression by SelfPowered Digital Vibration Control, Journal of Vibration and Acoustics, 134 (2012) 024502.

[14] S.J. Elliott, M. Zilletti, M. Ghandchi Tehrani, E. Rustighi, Maximising power absorption in a system for self-tuning structural damping and energy harvesting, in: EuroMech Colloquium 530: Structural Control and Energy Harvesting, Bristol, 2011.

[15] D. Niederberger, S. Behrens, A.J. Fleming, S.O.R. Moheimani, M. Morari, Adaptive electromagnetic shunt damping, Mechatronics, IEEE/ASME Transactions on, 11 (2006) 103-108.

[16] S.J. Elliott, M. Zilletti, Scaling of electromagnetic transducers for shunt damping and power harvesting, in: Proceedings of ISMA 2012 - USD 2012, Katholieke Universiteit Leuven, Belgium, 2012, pp. 463-474.

[17] A. Preumont, Mechatronics: Dynamics of Electromechanical and Piezoelectric Systems, Springer, Dordrecht, 2006.

\section{FIGURES}
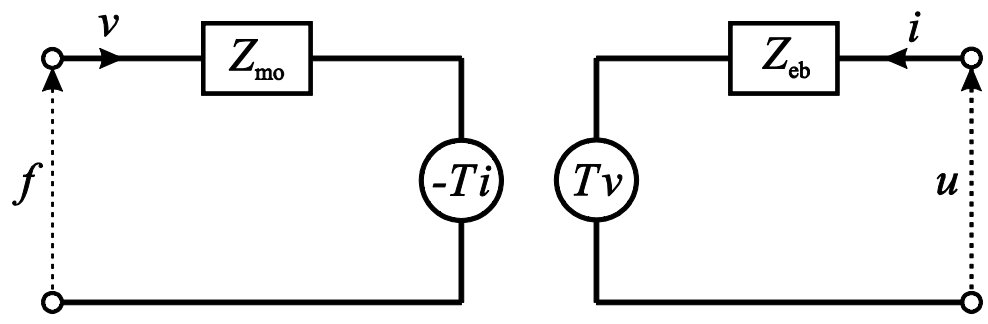

Figure 1: Block diagram of an electromagnetic transducer in terms of its two-port parameters

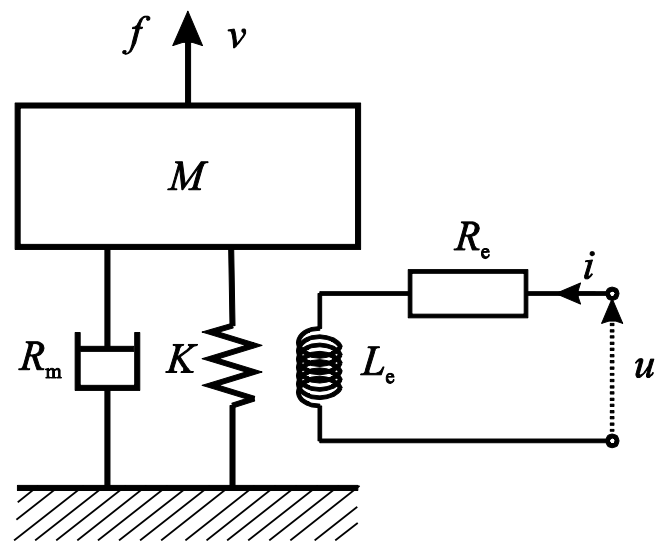

mass-driven

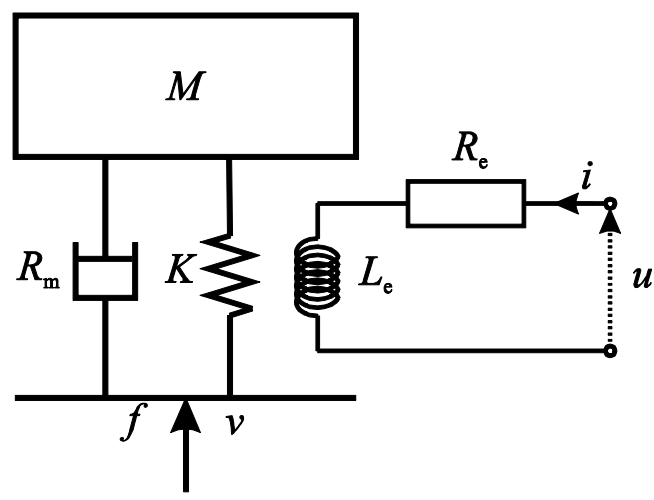

base-driven

Figure 2: Block diagram of an idealised electromagnetic actuator when driven by the moving mass, left, and when driven by the base structure, for an inertial device, right. 

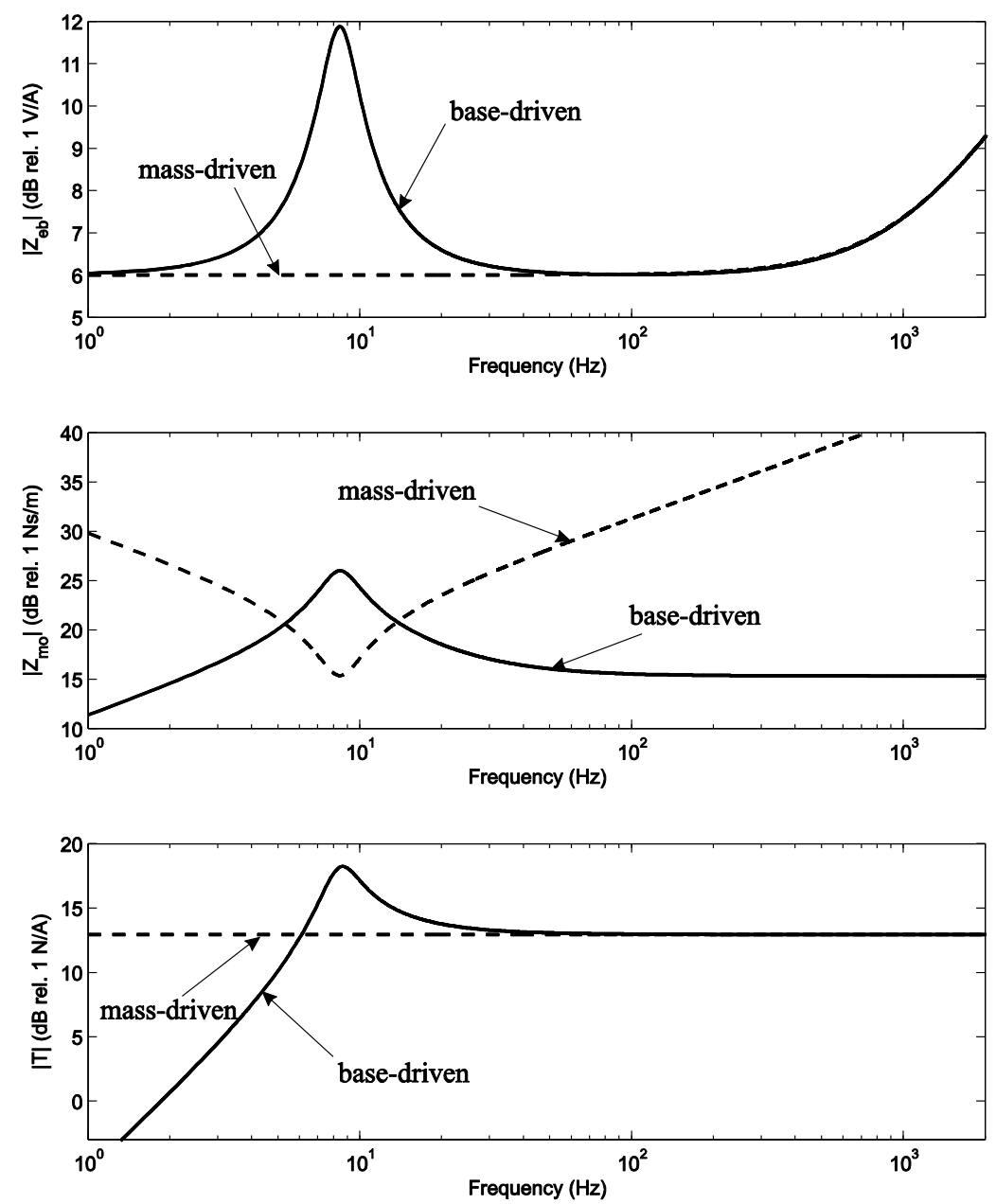

Figure 3: The modulus of the blocked electrical impedance $\left|Z_{e b}\right|$, open circuit impedance $\left|Z_{m o}\right|$ and transduction coefficient $|T|$ for an electromagnetic transducer with the force acting on the mass, mass-driven, dashed lines, and the force acting at the base, base-driven or inertial, solid lines, with the example parameters in the Table 1.
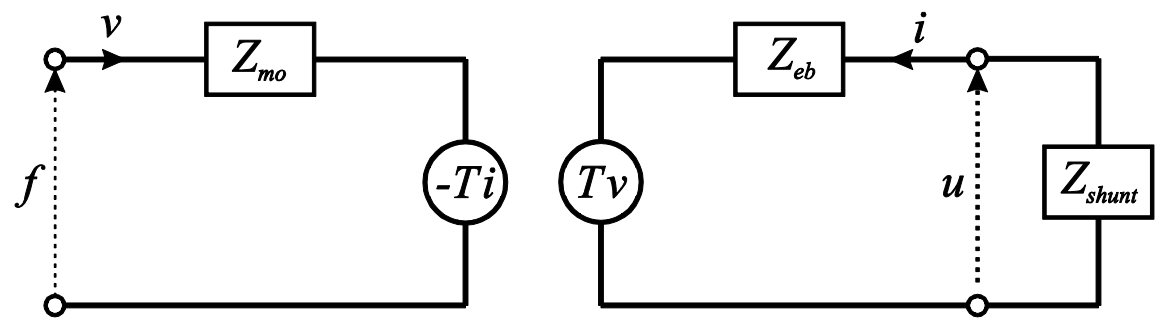

Figure 4: Block diagram of an electromagnetic transducer with a shunt impedance, $Z_{\text {shunt }}$, across its electrical terminals. 


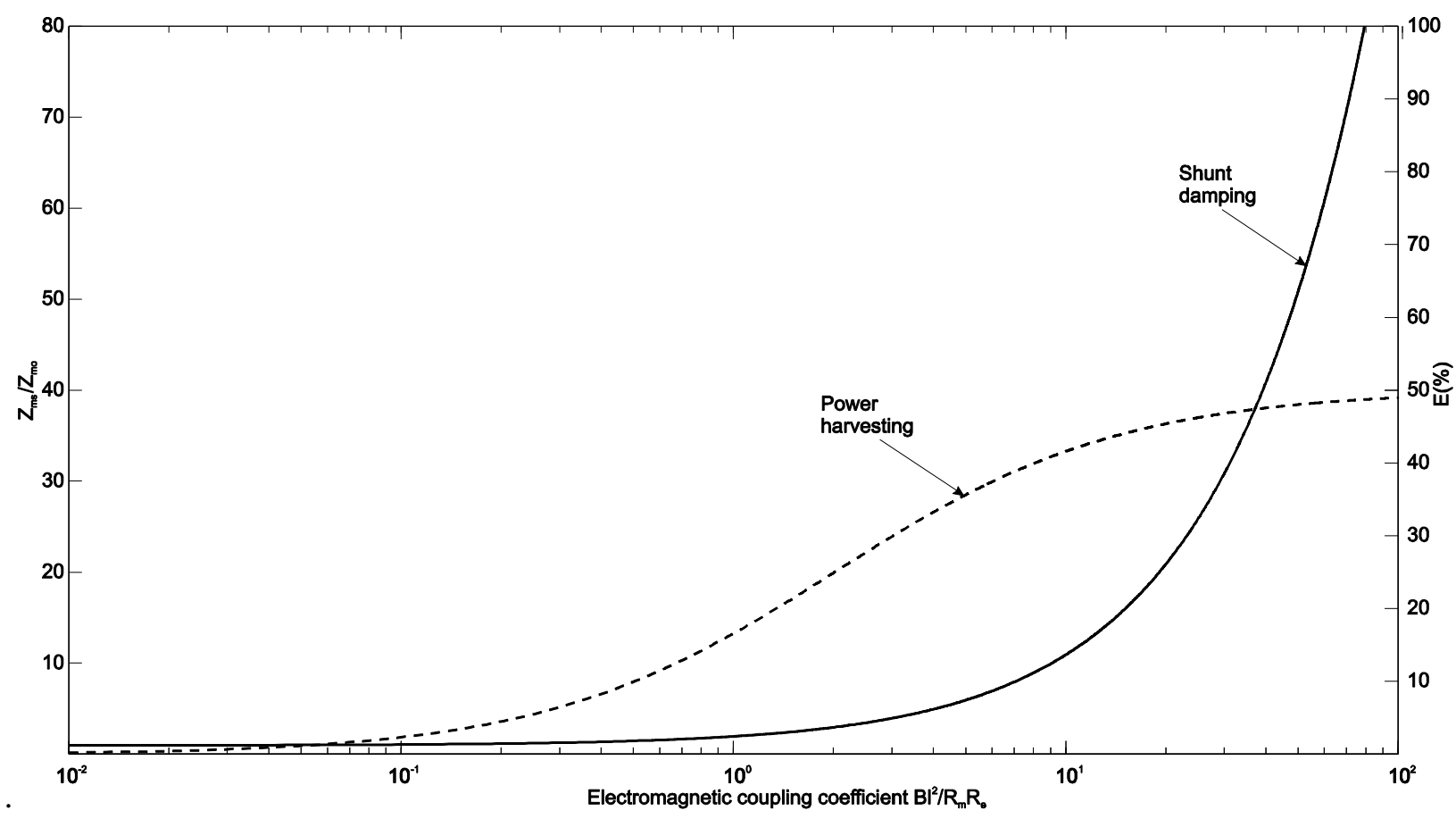

Figure 5: variation of the ratio of the electromagnetic transducer's mechanical impedance when short circuit and open circuit, $Z_{m s} / Z_{m o}$ (solid line) as function of the electromechanical coupling coefficient $C_{m e}=$ $(B l)^{2} / R_{m} R_{e}$, and the corresponding variation of the efficiency of the transducer as anenergy harvester $E$, given by the ratio of the electrical power harvested to the mechanical power supplied

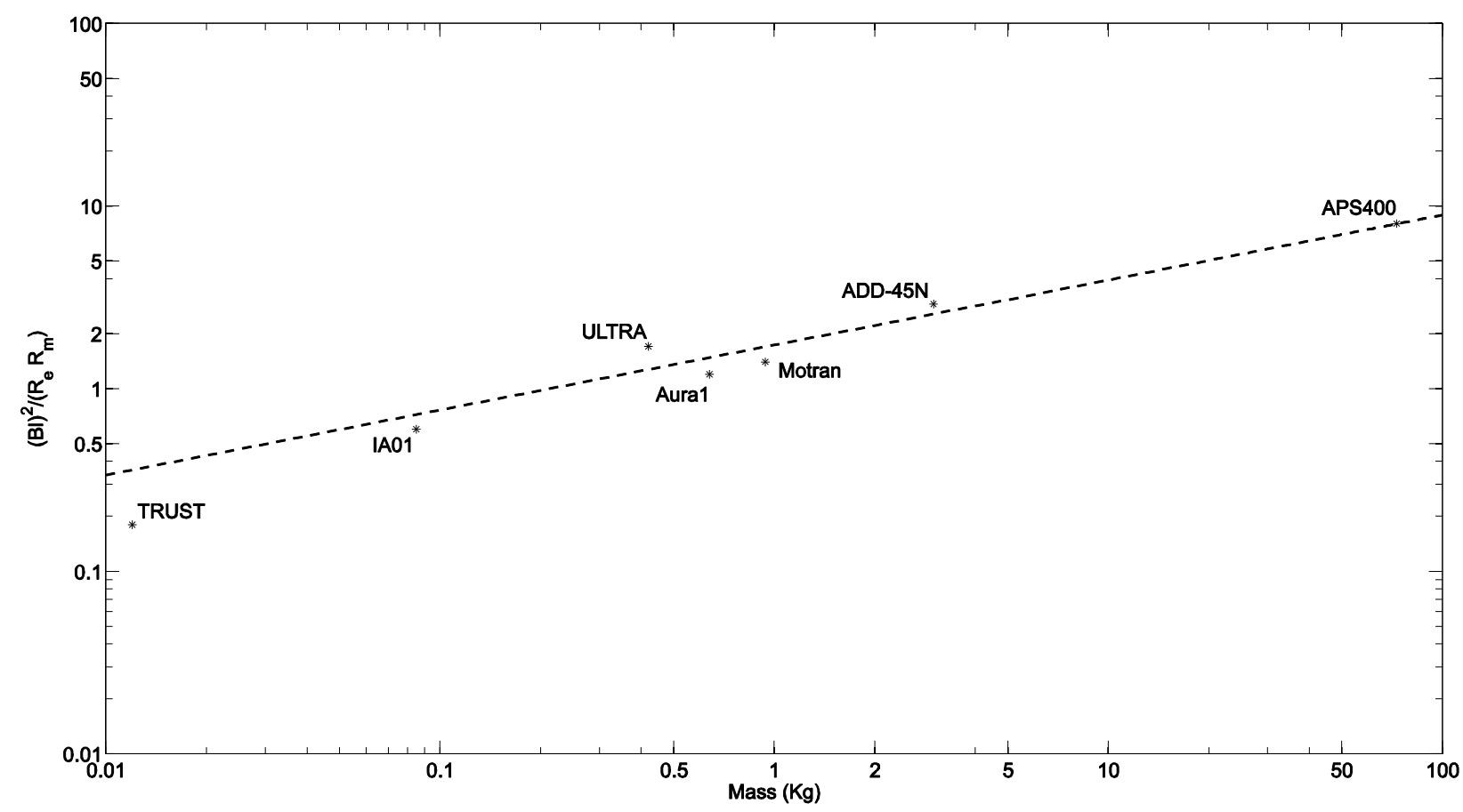

Figure 6: The log of the non-dimensional electromagnetic coupling coefficient, $(B l)^{2} / R_{e} R_{m}$, for different transducers, plotted as a function of the log of their mass. The best linear fit to this datais shown as the dashed line, which suggests that $(B l)^{2} / R_{e} R_{m}$ scales as mass to the power of about 0.35 . 


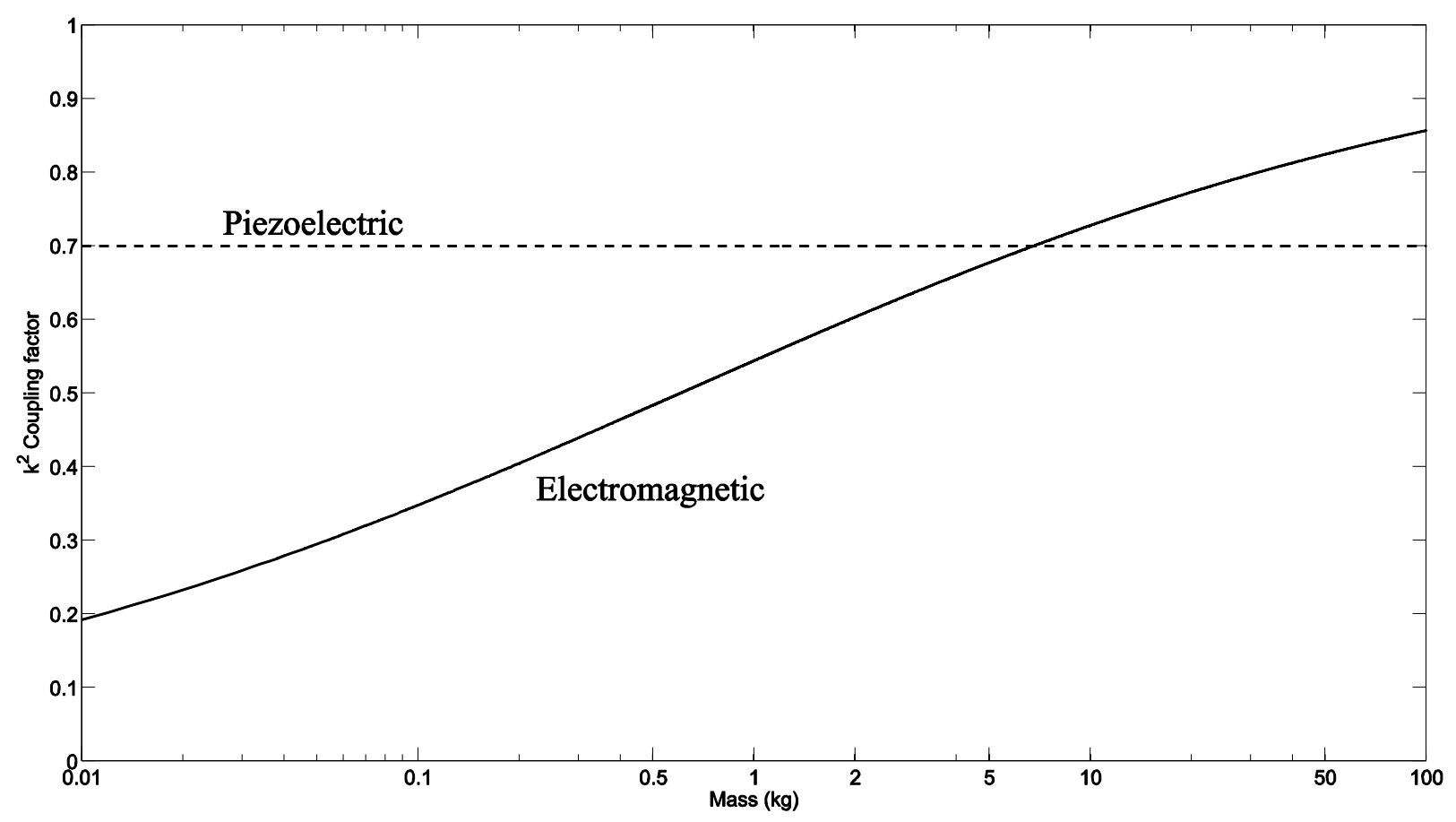

Figure 7: The effective electromechanical coupling factor as a function of the actuator mass for both electromagnetic transducers, using equation (25) and for piezoelectric transducers, assuming a PZT device.

\section{TABLES}

Table 1:Parameters of an example electromagnetic actuator, mostly taken from the datasheets for the Micromega Dynamics ADD-45N device

\begin{tabular}{l|l}
\hline \hline Moving mass $(M)$ & $2.2 \mathrm{~kg}$ \\
\hline Suspension Stiffness $(K)$ & $6130 \mathrm{Nm}^{-1}$ \\
\hline Suspension damping ratio $(\zeta)$ & 0.15 \\
\hline Suspension mechanical resistance $\left(R_{\mathrm{m}}\right)$ & $35 \mathrm{Nsm}^{-1}$ \\
\hline Electrical resistance $\left(R_{e}\right)$ & $4 \Omega$ \\
\hline Electrical inductance $\left(L_{\mathrm{e}}\right)$ & $0.6 \mathrm{mH}$ \\
\hline Force constant $(\mathrm{Bl})$ & $20 \mathrm{NA}^{-1}$ \\
\hline Total mass & $3 \mathrm{Kg}^{-}$ \\
\hline Peak force & $45 \mathrm{~N}$ \\
\hline Resonant frequency $\left(\omega_{n} / 2 \pi\right)$ & $8.4 \mathrm{~Hz}$ \\
\hline \hline
\end{tabular}


Table 2:The parameters of a number of electromagnetic inertial actuators

\begin{tabular}{l|c|c|c|c|c}
\hline \hline Type & $B l\left(\mathrm{NA}^{-1}\right)$ & $R_{\mathrm{e}}(\Omega)$ & $R_{\mathrm{m}}\left(\mathrm{Nsm}^{-1}\right)$ & $(B l)^{2} / R_{\mathrm{e}} R_{\mathrm{m}}$ & Total mass $(\mathrm{kg})$ \\
\hline \hline APS 400 & 37 & 3.0 & 53 & 8.0 & 73 \\
\hline Micromega (ADD-45N) & 20 & 4 & 35 & 2.9 & 3 \\
\hline Motran (IFY 30-100) & 10 & 1.6 & 44 & 1.4 & 0.94 \\
\hline Aura & 7 & 4.4 & 9 & 1.2 & 0.64 \\
\hline Ultra & 7.1 & 3.2 & 9.4 & 1.7 & 0.42 \\
\hline Micromega (IA-01) & 1.6 & 3.0 & 1.4 & 0.6 & 0.085 \\
\hline $\begin{array}{l}\text { TRUST headphone } \\
\text { actuator }\end{array}$ & 0.74 & 8 & 0.38 & 0.18 & 0.012 \\
\hline \hline
\end{tabular}

